\title{
Self-organized helical equilibria as a new paradigm for ohmically heated fusion plasmas
}

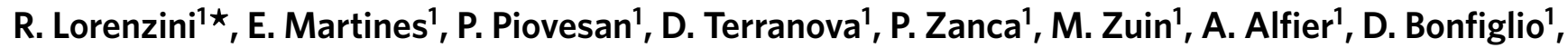

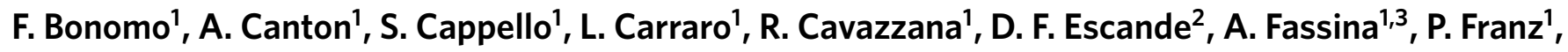 \\ M. Gobbin' ${ }^{1}$, P. Innocente', L. Marrelli', R. Pasqualotto ${ }^{1}$, M. E. Puiatti', M. Spolaore', M. Valisa', \\ N. Vianello', P. Martin ${ }^{1,3}$ and RFX-mod team and collaborators ${ }^{4}$
}

In the quest for new energy sources, the research on controlled thermonuclear fusion ${ }^{1}$ has been boosted by the start of the construction phase of the International Thermonuclear Experimental Reactor ${ }^{2}$ (ITER). ITER is based on the tokamak magnetic configuration ${ }^{3}$, which is the best performing one in terms of energy confinement. Alternative concepts are however actively researched, which in the long term could be considered for a second generation of reactors. Here, we show results concerning one of these configurations, the reversed-field pinch ${ }^{4,5}$ (RFP). By increasing the plasma current, a spontaneous transition to a helical equilibrium occurs, with a change of magnetic topology. Partially conserved magnetic flux surfaces emerge within residual magnetic chaos, resulting in the onset of a transport barrier. This is a structural change and sheds new light on the potential of the RFP as the basis for a low-magnetic-field ohmic fusion reactor.

The main magnetic field configurations studied for the confinement of toroidal fusion-relevant plasmas are the tokamak ${ }^{3}$, the stellarator $^{6}$ and the reversed-field pinch $^{4,5}$ (RFP). In the tokamak, a strong magnetic field is produced in the toroidal direction by a set of coils approximating a toroidal solenoid, and the poloidal field generated by a toroidal current flowing into the plasma gives the field lines a weak helical twist. This is the configuration that has been most studied and has achieved the best levels of energy confinement time. Thus, it is the natural choice for the International Thermonuclear Experimental Reactor, which has the mission of demonstrating the scientific and technical feasibility of controlled fusion with magnetic confinement.

The RFP, like the tokamak, is axisymmetric and exploits the pinch effect due to a current flowing in a plasma embedded in a toroidal magnetic field. The main difference is that, for a given plasma current, the toroidal magnetic field in a RFP is one order of magnitude smaller than in a tokamak, and is mainly generated by currents flowing in the plasma itself. This feature is underlying the main potential advantage of the RFP as a reactor concept, namely the capability of achieving fusion conditions with ohmic heating only in a much simpler and compact device. In the past, this positive feature was overcome by the poorer stability properties, which led to the growth and saturation of several magnetohydrodynamic (MHD) instabilities, eventually downgrading the confinement performance. These instabilities, represented by Fourier modes in the poloidal and toroidal angles $\theta$ and $\phi$ as $\exp [i(m \theta-n \phi)]$, with $m=1$ and $n>3 R / 2 a$ (ref. 7), were considered as an unavoidable ingredient of the dynamo self-organization process ${ }^{4,8,9}$, necessary for the sustainment of the configuration in time. The occurrence of several MHD modes resonating on different plasma layers gives rise to overlapping magnetic islands, which result in a chaotic region, extending over most of the plasma volume ${ }^{10}$, where the magnetic surfaces are destroyed and the confinement level is modest. This condition is generally dubbed as the multiple-helicity state.

An efficient strategy for chaos suppression in RFPs is based on inductive control of the current profile, which was successfully proven, although transiently ${ }^{11,12}$. A different strategy, potentially stationary and relying on self-organization, is based on the theoretical prediction that the RFP could exist in the chaos-free single-helicity condition, where only one of the core-resonant resistive modes would provide the dynamo effect ${ }^{13-15}$. To now, the best approximation of single helicity were quasi-singlehelicity (QSH) states: here, the innermost resonant $m=1$ mode dominates over the others, the so-called secondary modes, which maintain a finite amplitude. QSH states have been observed in several RFP devices ${ }^{5,16,17}$ and predicted in numerical simulations ${ }^{18}$. They are an intermediate state between the multiple-helicity and the theoretical single-helicity ones, because the secondary modes are still present.

Here, we present experimental findings obtained in the RFXmod device ${ }^{19}$ that show a clear evolution of the plasma towards the single-helicity state. This has been made possible by the exploration of a wide range of plasma current levels, which for the first time in a RFP have reached values higher than $1 \mathrm{MA}$, and by the control of the radial magnetic field at the plasma boundary through an advanced feedback system ${ }^{20}$. The novel results are that, as the current is raised, the plasma shows an increasing preference for the QSH state, and that this state becomes purer (the normalized secondary mode amplitude is reduced). Furthermore, above a threshold of about $4 \%$ in the dominant mode normalized amplitude, a topological change in the magnetic configuration is observed: the separatrix X-point of the dominant mode magnetic island merges with the main magnetic axis, and the two disappear. The former island $\mathrm{O}$-point becomes the only magnetic axis and a helical plasma column is obtained in an axisymmetric device. This new single-helical-axis (SHAx) state ${ }^{21}$ is theoretically predicted ${ }^{22}$ to be more resilient to the magnetic chaos induced by the secondary modes.

Here, we show that this new equilibrium features strong electron transport barriers ${ }^{21}$, consistent with the mean helical magnetic

${ }^{1}$ Consorzio RFX, Associazione Euratom-ENEA sulla Fusione, Padova 35137, Italy, ${ }^{2}$ Physique des Interactions loniques et Moléculaires, UMR 6633-CNRS/Aix Marseille Université, France, ${ }^{3}$ Dipartimento di Fisica 'G. Galilei', Università di Padova, Padova 35131, Italy, ${ }^{4}$ See end of paper for details. *e-mail: rita.lorenzini@igi.cnr.it. 


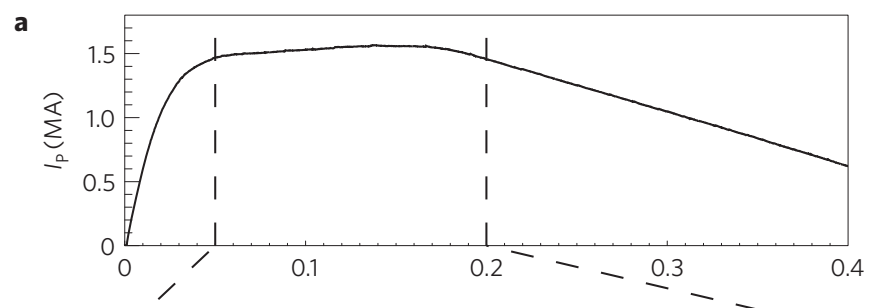

b

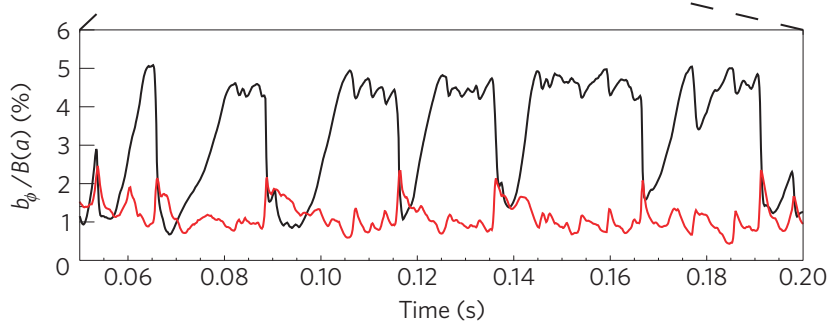

Figure 1 | Typical 1.5 MA plasma discharge in RFX-mod. a, Plasma current as a function of time. The vertical dashed lines delimit the so-called flat-top phase of the discharge. $\mathbf{b}$, The black curve shows the amplitude of the $m=1 / n=7$ dominant mode during the flat-top phase of the discharge, and the red curve shows the amplitude of the secondary modes $(m=1 / n=8-23)$, defined as the square root of the sum of their squared amplitudes. The amplitudes are those of the toroidal magnetic field component at the plasma surface, as obtained with a Fourier transform in space of the signals given by a system of $48 \times 4$ pick-up coils located outside the vacuum vessel, and are normalized to the average poloidal magnetic field measured at the same location. It is clearly seen that the system oscillates between two states, one where all of the modes have similar amplitudes (multiple helicity) and another one where there are a dominant mode and secondary ones (QSH).

configuration associated with the dominant mode. The SHAx state is characterized by the emergence of almost invariant magnetic surfaces, although in the presence of a small residual magnetic chaos, giving rise to improved confinement properties. These surfaces are interpreted as ghost surfaces ${ }^{23,24}$.

Figure la shows a plasma current waveform for a $1.5 \mathrm{MA}$ discharge carried out in RFX-mod. The amplitude of the innermost resonant $m=1$ mode $(n=7)$ and the total amplitude of the secondary $m=1$ modes are shown, for the flat-top phase, in Fig. 1b. The graph shows that the plasma preferentially stays in a QSH state where the $n=7$ mode is dominant and the other modes are reduced. These phases can last ten times longer than the typical energy confinement time $(\sim 3 \mathrm{~ms})$. Occasional backtransitions to the multiple-helicity state occur, as observed also in numerical simulations ${ }^{18}$. In the QSH phases, the normalized edge amplitude of the dominant mode reaches values of about $4 \%$, and a SHAx state is obtained.

The QSH properties are found to be related to the Lundquist number $S$, a dimensionless parameter relevant to the MHD dynamics, defined as the ratio of the resistive diffusion time to the Alfvén time ${ }^{3}$. Figure $2 \mathrm{a}$ shows the dependence of the QSH persistence with $S$, showing that higher $S$ values, which correspond to hotter plasmas obtained at higher current levels, lead to longer persistence, reaching values up to $90 \%$. The amplitude of the dominant and secondary modes during the QSH phase is also found to depend on the Lundquist number. This is shown in Fig. 2b, from which it is apparent that secondary modes decrease steadily as $S$ rises, whereas the dominant one shows at first a fast rise, then followed by a slower increase. As the transition to a SHAx state occurs when a threshold in the dominant mode amplitude is exceeded, this implies that at high $S$ the QSH states are indeed SHAx. It is important to remark that the increase of $S$ shown in Fig. 2 is due to the increase of the flat-top current and

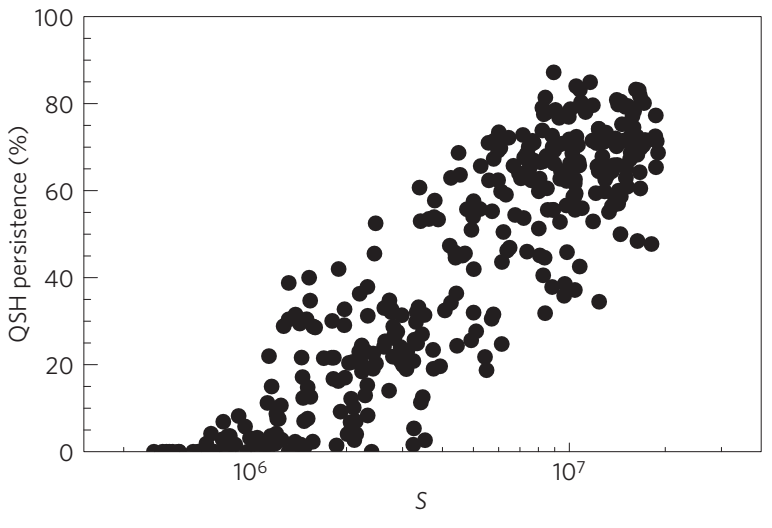

b

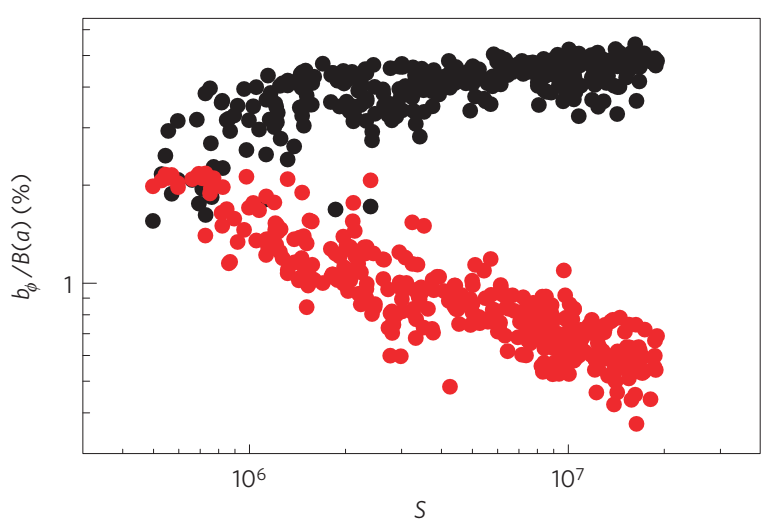

Figure 2 | Mode amplitudes versus the Lundquist number. QSH properties plotted versus the Lundquist number $S$ for a wide database of discharges with currents ranging between 0.3 and 1.6 MA. a, QSH persistence, quantified as the ratio between the total duration of the QSH during the current flat-top and the flat-top duration, plotted as a function of $S$. The persistence is longer at higher $\mathrm{S}$. $\mathbf{b}$, Amplitude of the dominant (black) and secondary (red) modes during the QSH phase plotted as a function of $S$. As the Lundquist number increases, the dominant mode amplitude grows, whereas the amplitude of the secondary ones is reduced.

the consequent rise in temperature, and that no extra sources of plasma heating are used.

To assess the quality of magnetic equilibrium, we have developed a simple, yet effective method for computing the helical flux $\chi(\mathbf{r})$ and therefore the position and shape of magnetic surfaces for the SHAx state. When these surfaces are not destroyed by magnetic chaos, flux functions (that is, any function $f(\mathbf{r})$ such that $\mathbf{B} \cdot \nabla f=0$ ) are constant on them ${ }^{1}$. In particular, this applies to kinetic pressure and, owing to the fast parallel transport, to temperature and density. A typical electron temperature profile measured in a SHAx state is shown in Fig. 3a. Figure $3 \mathrm{~b}$ shows the same profile plotted as a function of the effective radius $\rho=\left(\chi / \chi_{0}\right)^{1 / 2}$, where $\chi_{0}$ is the helical flux at the plasma boundary. It can be seen that the two half-profiles measured on the two sides of the magnetic axis, marked by different colours, collapse onto each other only when plotted as a function of $\rho$. In particular, the strong gradients marked by the shaded region, which indicate the formation of a transport barrier, turn out to be the same, showing that they appear different in cylindrical coordinates only because of the different spacing of the flux surfaces on the two sides. A reconstruction of the two-dimensional (2D) temperature map on the poloidal plane is shown in Fig. 3c. The hot region is bean-shaped, and centred off the geometric axis, on the resonant surface of the $m=1 / n=7$ mode.

These results are further confirmed by the high-space-resolution soft X-ray (SXR) tomographic diagnostic: SXR emissivity is, in 

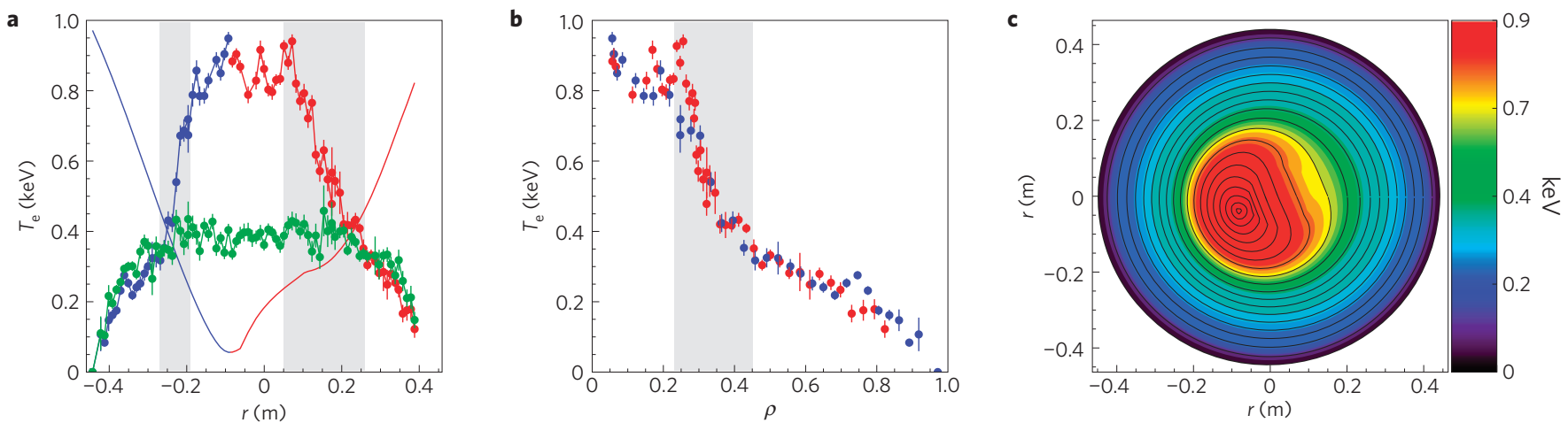

Figure 3 | Mapping of the temperature profile on the helical flux surfaces. The electron temperature at a given time is measured in 84 points aligned along a horizontal diameter of the vacuum vessel with the Thomson scattering technique ${ }^{28}$. a, Typical electron temperature profile measured in $\sim 1.5 \mathrm{MA}$ discharges when the plasma is in a SHAx state. It can be seen that a high-temperature $(\sim 0.8-0.9 \mathrm{keV})$ region is present in the core plasma and extends on both sides of the geometric axis, a typical feature of SHAx states. The profile is asymmetric with respect to the geometric axis, and the strong gradient regions (shaded) show substantially different gradients on the two sides. On the same graph, the continuous line gives the profile of the effective radial coordinate $\rho$. Red and blue refer to the two opposite sides with respect to the helical magnetic axis. A typical profile in multiple helicity is shown in green for reference. $\mathbf{b}$, Temperature profile plotted as a function of $\rho$, showing the collapse of the two half-profiles one onto the other. The error bars in $\mathbf{a}$ and $\mathbf{b}$ are given by the confidence limit on the temperature resulting from the gaussian fit of the scattered light spectrum. c, Reconstruction of the full $2 \mathrm{D}$ map of the temperature on the poloidal plane, obtained from the dependence of $\chi$ on the geometric coordinates. The $\chi$ contour lines are also shown.

a

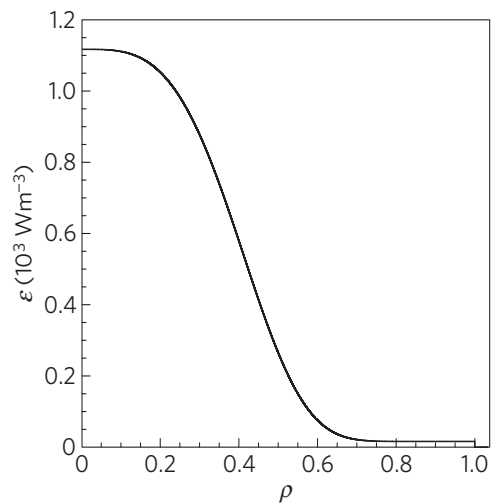

d

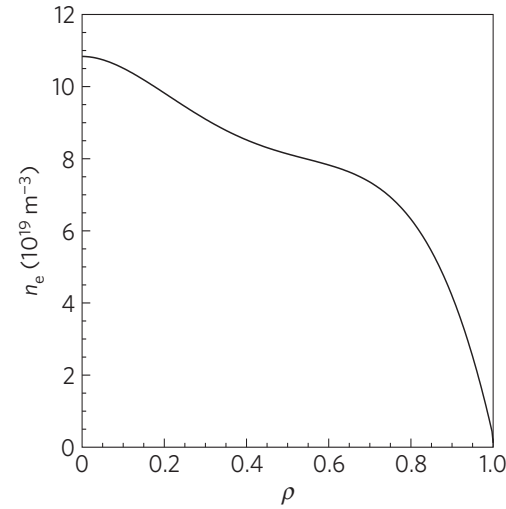

b
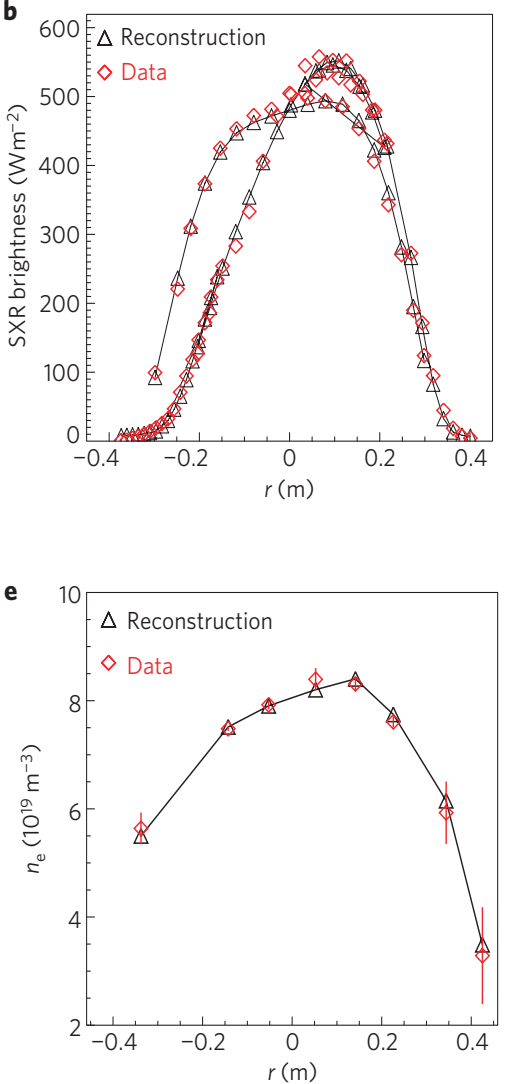

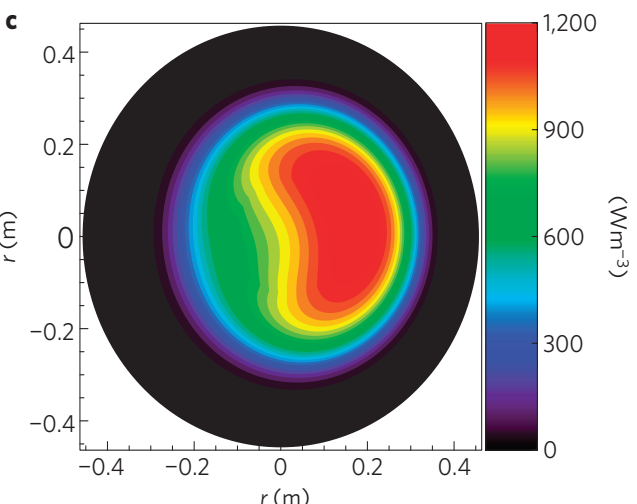

f

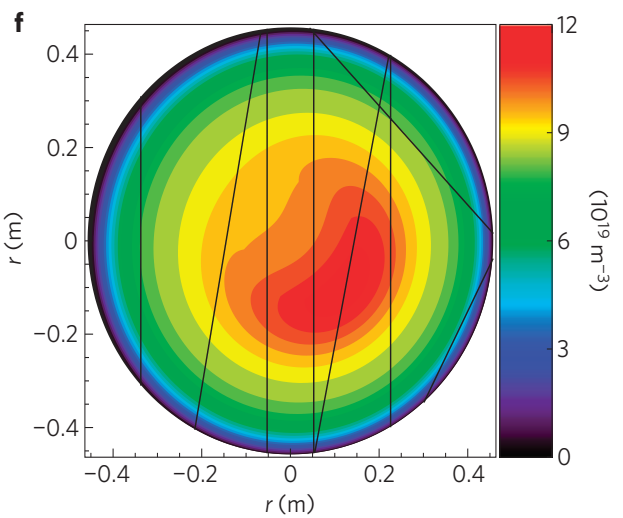

Figure 4 | Mapping of line-integrated emissivity and line-averaged density measurements on helical flux surfaces. The $X$-ray emissivity is measured by silicon photodiode sensors ${ }^{29}$ along 78 lines of sight organized in two sets, one nearly horizontal and one nearly vertical, lying on the same poloidal plane. Each measurement is a line integral along the corresponding chord giving the SXR brightness. The plasma density is measured along 8 chords by an interferometric technique ${ }^{30}$. Each measurement is a line integral along the corresponding chord. a, Emissivity profile parametrized using a simple three-parameter model of the form $\varepsilon(\rho)=\varepsilon_{0}\left(1-\rho^{\alpha}\right)^{\beta}$. b. Measurements of line-averaged X-ray emissivity in red and values reconstructed using the $\varepsilon$ profile in $\mathbf{a}$ in black. An excellent match between measurements and reconstructed values can be observed. $\mathbf{c}, 2 \mathrm{D}$ emissivity map resulting from the reconstructions. $\mathbf{d}$, Density profile parametrized using a model of the form $n(\rho)=a+b \rho^{2}+c \rho^{3}+d \rho^{4}$. e, Line-averaged density values measured by the interferometric system during a pellet injection and values reconstructed using the profile in $\mathbf{d}$. The error bars are given by the standard deviations calculated at the end of the discharge. f, Reconstructed 2D density map. The straight lines represent the chords along which measurements are made. 
fact, a strongly increasing function of the electron temperature. By assuming the SXR emissivity to be a simple function of the helical flux (as shown in Fig. 4a) and properly tuning the $\rho$ dependence, it has been possible to well reproduce the lineintegrated measurements (Fig. 4b). The 2D emissivity map (Fig. 4c) shows once again that the hot core region is bean-shaped.

Finally, the same approach has been applied to electron density measurement. Owing to the small particle source in the plasma core, being the plasma fuelled at the edge by wall recycling, the density profile inside the core is nearly flat ${ }^{25}$. To induce a density gradient in the core, solid hydrogen pellets ${ }^{26}$ have been launched into the plasma, so that their trajectories would pass through the centre of the bean-shaped region in SHAx states. One such case is illustrated in Fig. 4e, where measurements of chord-averaged plasma density are shown at the time when pellet ablation ends. The asymmetric profile can be explained only by a dependence of the density on the helical flux, as previously done for the electron temperature and SXR emissivity profiles. An analysis similar to that carried out for the tomographic data has been carried out. The reconstructed profile and the $2 \mathrm{D}$ density pattern are respectively shown in Fig. 4d and $\mathrm{f}$.

The available ion temperature measurements (determined from Doppler broadening of the spectral line emitted by the $\mathrm{H}$-like $\mathrm{O}^{7+}$ ion) are not sufficiently resolved in space and time to assess whether an ion transport barrier is also formed. The measured line-averaged ion temperature, ranging from approximately 0.5 to 0.7 of the electron temperature, is consistent with a classical collisional electron-ion energy transfer.

On the basis of the results shown in Figs 3 and 4, the assumption of good isobaric and isoemissive helical flux surfaces-a signature of MHD equilibrium-allows a consistent interpretation of the data. Although residual magnetic chaos is present, these good-quality surfaces lead to robust electron temperature gradients and are the likely signature of ghost surfaces at constant $\chi$.

The spontaneous occurrence of a new self-organized helical equilibrium with a single helical axis, reduced magnetic fluctuations and strong transport barriers provides a change of paradigm for the RFP. As the persistence of these improved confinement phases increases with plasma current, the likelihood of achieving steady SHAx states in multi-mega-ampere devices can be inferred. This opens a promising path for this configuration, because the emergence of ghost surfaces is likely to be followed, at even lower secondary mode amplitudes, by the separation of the magnetic islands. Island separation would lead to the formation of regions of conserved surfaces, giving rise to transitions to further improved confinement regimes. Further improvements are also expected by optimization of magnetic boundary and fuelling, the latter corroborated by preliminary experiments with pellet injection. This new vision supports a reappraisal of the RFP as a low-external-field, non-disruptive, ohmically heated approach to magnetic fusion, exploiting both self-organization and technological simplicity. The RFP is thus confirmed as a flexible environment for developing fusion science and for contributing to fusion predictive capabilities.

\section{Methods}

The flux surfaces in the SHAx state have been computed assuming that the magnetic field is the superposition of an axisymmetric equilibrium and of the helical perturbation corresponding to the dominant $m=1$ mode eigenfunction. This eigenfunction is computed by means of the toroidal force-free Newcomb's equation, supplemented by magnetic measurements at the plasma edge used as boundary conditions ${ }^{27}$. The helical flux $\chi(\mathbf{r})$ is given by $\chi=m \Psi_{0}-n F_{0}+\left(m \psi^{m n}-n f^{m n}\right) \exp [i(m \vartheta-n \varphi)]+$ c.c., where $\Psi_{0}$ and $F_{0}$ are the poloidal and toroidal fluxes of the axisymmetric equilibrium, $\psi^{m n}$ and $f^{m n}$ are those of the dominant mode and $\vartheta$ and $\varphi$ are the flux coordinates defined in ref. 27. It can be shown that $\chi$ is constant along magnetic field lines (that is, $\mathbf{B} \cdot \nabla \chi=0)$. Therefore, its contour plots represent the nested helical flux surfaces of the SHAx state.
Received 19 December 2008; accepted 18 May 2009; published online 14 June 2009

\section{References}

1. Freidberg, J. Plasma Physics and Fusion Energy (Cambridge Univ. Press, 2007).

2. The ITER physics basis. Nucl. Fusion 47, S1-S413 (2007).

3. Wesson, J. Tokamaks 3rd edn (Oxford Univ. Press, 2004).

4. Ortolani, S. \& Schnack, D. D. Magnetohydrodynamics of Plasma Relaxation (World Scientific, 1993).

5. Martin, P. et al. Overview of quasi-single helicity experiments in reversed field pinches. Nucl. Fusion 43, 1855-1862 (2003).

6. Boozer, A. H. What is a stellarator? Phys. Plasmas 5, 1647-1655 (1998).

7. Antoni, V., Martin, P. \& Ortolani, S. Experimental evidence of on axis q oscillations in Eta Beta II. Plasma Phys. Control. Fusion 29, 279-285 (1987).

8. Ji, H. \& Prager, S. C. The alpha dynamo effects in laboratory plasmas. Magnetohydrodynamics 18, 191-210 (2002).

9. Ji, H., Almagri, A. F., Prager, S. C. \& Sarff, J. S. Time resolved observation of discrete and continuous magnetohydrodynamic dynamo in the reversed-field pinch edge. Phys. Rev. Lett. 73, 668-671 (1994).

10. Paccagnella, R. \& D'Angelo, F. The stochastic diffusion process in reversed-field pinch. Phys. Plasmas 3, 2353-2364 (1996).

11. Sarff, J. S., Hokin, S. A., Ji, H., Prager, S. C. \& Sovinec, C. R. Fluctuation and transport reduction in a reversed field pinch by inductive poloidal current drive. Phys. Rev. Lett. 72, 3670-3673 (1994).

12. Chapman, B. E. et al. Improved confinement plasmas at high temperature and high beta in the MST RFP. Nucl. Fusion (in the press).

13. Cappello, S. \& Paccagnella, R. in Proc. of the Workshop on Theory of Fusion Plasmas (ed. Sindoni, E.) 595-604 (1990).

14. Cappello, S. \& Paccagnella, R. Nonlinear plasma evolution and sustainment in the reversed field pinch. Phys. Fluids B 4, 611-618 (1992).

15. Finn, J. M., Nebel, R. \& Bathke, C. Single and multiple helicity ohmic states in reversed field pinches. Phys. Fluids B 4, 1262-1279 (1992).

16. Escande, D. F. et al. Quasi-single-helicity reversed-field-pinch plasmas. Phys. Rev. Lett. 85, 1662-1665 (2000).

17. Martin, P. Magnetic and thermal relaxation in the RFP. Plasma Phys. Control. Fusion 41, A247-A255 (1999).

18. Cappello, S. \& Escande, D. F. Bifurcation in viscoresistive MHD: The Hartmann number and the reversed field pinch. Phys. Rev. Lett. 85, 3838-3841 (2000).

19. Sonato, P. et al. Machine modification for active MHD control in RFX Fusion Eng. Des. 66-68, 161-168 (2003).

20. Zanca, P., Marrelli, L., Manduchi, G. \& Marchiori, G. Beyond the intelligent shell concept: The clean-mode-control. Nucl. Fusion 47, 1425-1436 (2007).

21. Lorenzini, R. et al. Single-helical axis states in reversed-field-pinch plasmas. Phys. Rev. Lett. 101, 025005 (2008).

22. Escande, D. F. et al. Chaos healing by separatrix disappearance and quasisingle helicity states of the reversed field pinch. Phys. Rev. Lett. 85, 3169-3172 (2000).

23. Golé, C. Ghost circles for twist maps. J. Differ. Equ. 97, 140-173 (1992).

24. Hudson, S. R. \& Breslau, J. Temperature contours and ghost surfaces for chaotic magnetic fields. Phys. Rev. Lett. 100, 095001 (2008).

25. Bonomo, F. et al. 2D characterization of thermal core topology changes in controlled RFX-mod QSH states. Nucl. Fusion 49, 045011 (2009).

26. Baker, W. et al. Proc. 16th IEEE/NPSS Symp. on Fusion Engineering Vol. 2, 1570-1573 (1995)

27. Zanca, P. \& Terranova, D. Reconstruction of the magnetic perturbation in a toroidal reversed field pinch. Plasma Phys. Control. Fusion 46, 1115-1141 (2004).

28. Alfier, A. \& Pasqualotto, R. New Thomson scattering diagnostic on RFX-mod. Rev. Sci. Instrum. 78, 013505 (2007).

29. Franz, P. et al. Soft $X$ ray tomographic imaging in the RFX reversed field pinch. Nucl. Fusion 41, 695-709 (2001).

30. Innocente, P. et al. Upgrade of the RFX $\mathrm{CO}_{2}$ interferometer using in-vessel optics for extended edge resolution. Rev. Sci. Instrum. 68, 694-697 (1997).

\section{Acknowledgements}

We thank the whole Consorzio RFX staff for the support in the device operation. This work has been supported by the European Communities under the contract of Association between EURATOM and ENEA.

\section{Author contributions}

The experiments were carried out by the whole RFX team, R.L., E.M., P.P., D.T., P.Z., M.Z. jointly conceived this paper, in close collaboration with A.A., D.B., F.B., A.C., S.C., L.C., R.C., D.F.E., A.F., P.F., M.G., P.I., L.M., R.P., M.S., M.V. and N.V. and with M.E.P. and P.M. who lead the RFX science programme.

\section{Additional information}

Reprints and permissions information is available online at http://npg.nature.com/ reprintsandpermissions. Correspondence and requests for materials should be addressed to R.L. 
The RFX-mod home team and collaborators, as in the 22nd IAEA Fusion Energy Conference, 13-18 October 2008, Geneva, Switzerland (overview OV/5-2Ra):

P. Martin ${ }^{5}$, L. Apolloni ${ }^{5}$, M. E. Puiatti ${ }^{5}$, J. Adamek ${ }^{6}$, M. Agostini ${ }^{5}$, A. Alfier ${ }^{5}$, S. V. Annibaldi ${ }^{7}$, V. Antoni ${ }^{5}$, F. Auriemma ${ }^{5}$, O. Barana ${ }^{5}$, M. Baruzzo ${ }^{5}$, P. Bettini ${ }^{5}$, T. Bolzonella ${ }^{5}$, D. Bonfiglio ${ }^{5}$, F. Bonomo ${ }^{5}$, M. Brombin ${ }^{5}$, J. Brotankova ${ }^{6}$, A. Buffa ${ }^{5}$, P. Buratti ${ }^{7}$, A. Canton ${ }^{5}$, S. Cappello ${ }^{5}$, L. Carraro ${ }^{5}$, R. Cavazzana ${ }^{5}$, M. Cavinato ${ }^{5}$, B. E. Chapman ${ }^{8}$, G. Chitarin ${ }^{5}$, S. Dal Bello ${ }^{5}$, A. De Lorenzi ${ }^{5}$, G. De Masi ${ }^{5}$, D. F. Escande ${ }^{5,9}$, A. Fassina ${ }^{5}$, A. Ferro ${ }^{5}$, P. Franz ${ }^{5}$, E. Gaio ${ }^{5}$, E. Gazza ${ }^{5}$, L. Giudicotti ${ }^{5}$, F. Gnesotto ${ }^{5}$, M. Gobbin ${ }^{5}$, L. Grando ${ }^{5}$, L. Guazzotto ${ }^{5}$, S. C. Guo ${ }^{5}$, V. Igochine ${ }^{10}$, P. Innocente ${ }^{5}$, Y. Q. Liu' ${ }^{11}$, R. Lorenzini ${ }^{5}$, A. Luchetta ${ }^{5}$, G. Manduchi ${ }^{5}$, G. Marchiori ${ }^{5}$, D. Marcuzzi ${ }^{5}$, L. Marrelli ${ }^{5}$, S. Martini ${ }^{5}$, E. Martines ${ }^{5}$, K. McCollam $^{8}$, F. Milani ${ }^{5}$, M. Moresco ${ }^{5}$, L. Novello ${ }^{5}$, S. Ortolani ${ }^{5}$, R. Paccagnella ${ }^{5}$, R. Pasqualotto ${ }^{5}$, S. Peruzzo ${ }^{5}$, R. Piovan ${ }^{5}$, P. Piovesan ${ }^{5}$, L. Piron ${ }^{5}$, A. Pizzimenti ${ }^{5}$, N. Pomaro ${ }^{5}$, I. Predebon ${ }^{5}$, J. A. Reusch ${ }^{8}$, G. Rostagni ${ }^{5}$, G. Rubinacci' ${ }^{12}$, J. S. Sarff ${ }^{8}$, F. Sattin ${ }^{5}$, P. Scarin ${ }^{5}$, G. Serianni ${ }^{5}$, P. Sonato ${ }^{5}$, E. Spada ${ }^{5}$, A. Soppelsa ${ }^{5}$, S. Spagnolo ${ }^{5}$, M. Spolaore ${ }^{5}$, G. Spizzo ${ }^{5}$, C. Taliercio ${ }^{5}$, D. Terranova ${ }^{5}$, V. Toigo ${ }^{5}$, M. Valisa ${ }^{5}$, N. Vianello ${ }^{5}$, F. Villone ${ }^{13}$, R. B. White ${ }^{14}$, D. Yadikin ${ }^{10}$, P. Zaccaria ${ }^{5}$, A. Zamengo ${ }^{5}$, P. Zanca ${ }^{5}$, B. Zaniol ${ }^{5}$, L. Zanotto ${ }^{5}$, E. Zilli' ${ }^{5}$, H. Zohm ${ }^{10}$ and M. Zuin ${ }^{5}$

${ }^{5}$ Consorzio RFX, Associazione EURATOM-ENEA sulla Fusione, 35137 Padova, Italy, ${ }^{6}$ Institute of Plasma Physics, Association EURATOM-IPP.CR, Prague 18200, Czech Republic, ${ }^{7}$ Space and Plasma Physics, EE KTH, SE-10044 Stockholm, Sweden, ${ }^{8}$ Department of Physics, University of Wisconsin, Madison, Wisconsin 53706, USA, ${ }^{9}$ UMR 6633 CNRS-Université de Provence, Marseille, France, ${ }^{10}$ Max-Planck-Institut für Plasmaphysik, EURATOM Association, 85748 Garching, Germany, ${ }^{11}$ EURATOM-UKAEA Fusion Ass., Culham Science Centre, Abingdon OX14 3DB, UK, 12 Ass. Euratom/ENEA/CREATE, DIEL, Università di Napoli Federico II, Napoli 80125, Italy, ${ }^{13}$ Ass. Euratom/ENEA/CREATE, DAEIMI, Università di Cassino, Cassino 03043, Italy, ${ }^{14}$ Plasma Physics Laboratory, Princeton University, Princeton, New Jersey 08543, USA. 